\title{
Pricing Flexible Demand Non-Convexities in Electricity Markets
}

\author{
Yujian Ye, Dimitrios Papadaskalopoulos, and Goran Strbac \\ Department of Electrical and Electronic Engineering \\ Imperial College London \\ London, UK \\ yujian.ye11@imperial.ac.uk, d.papadaskalopoulos08@imperial.ac.uk, g.strbac@imperial.ac.uk
}

\begin{abstract}
Uniform marginal prices cannot generally support competitive equilibrium solutions in markets with nonconvexities and yield schedules' inconsistency and surplus suboptimality effects. Previous work has identified non-convexities associated with the generation side of electricity markets and proposed a generalized uplift approach to eliminate these effects. This paper examines the above issues from the perspective of the flexible demand (FD) side. FD non-convexities are identified, including its ability to forgo demand activities and minimum power levels, and resulting inconsistency and surplus suboptimality effects are demonstrated through simple examples. Generalized uplift functions for FD participants are proposed, including quadratic pricing terms to limit their tendency to concentrate at the lowest-priced periods, and binary terms associated with their ability to forgo activities. Finally, a new rule is proposed for the equitable distribution of the total surplus loss among the market participants. These contributions are supported by case studies on a market with a day-ahead horizon and hourly resolution.
\end{abstract}

Keywords-Competitive equilibrium, electricity markets, flexible demand, generalized uplifts, surplus sub-optimality.

\section{NOMENCLATURE}

A. Indices and Sets

$t \in T \quad$ Index and set of time periods

$i \in I \quad$ Index and set of generators

$j \in J \quad$ Index and set of FDs

$r \quad$ Index of iterations

$\mathcal{G}_{i} \quad$ Operating constraints set of generator $i$

$\mathcal{D}_{j} \quad$ Operating constraints set of FD $j$

$\Xi_{i} \quad$ Profit cutting planes set of generator $i$

$\Psi_{j} \quad$ Utility cutting planes set of FD $j$

\section{B. Variables}

$\lambda \quad$ Vector of electricity prices $\lambda_{t}(£ / M W h)$

$\boldsymbol{g}_{\boldsymbol{i}} \quad$ Vector of power outputs $g_{i t}$ of generator $i$ (MW)

$\boldsymbol{u}_{\boldsymbol{i}} \quad$ Vector of commitment statuses $u_{i t}$ of generator $i$

$\boldsymbol{d}_{\boldsymbol{j}} \quad$ Vector of power demands $d_{j t}$ of FD $j(\mathrm{MW})$ $v_{j} \quad$ Binary variable expressing whether the activity of FD $j$ is forgone ( $v_{j}=0$ if it is forgone, $v_{j}=1$ otherwise)

$\boldsymbol{w}_{\boldsymbol{j}} \quad$ Vector of binary variables $w_{j t}$ expressing whether FD $j$ is active at $t\left(w_{j t}=1\right.$ if it is active, $w_{j t}=0$ otherwise)

$\Delta \boldsymbol{\alpha}_{\boldsymbol{i}}^{\boldsymbol{g}} \quad$ Vector of uplift parameters $\Delta \alpha_{i t}^{g}$ associated with the power output of generator $i$ at $t(£ / M W h)$

$\Delta \boldsymbol{c}_{\boldsymbol{i}}^{\boldsymbol{o n}} \quad$ Vector of uplift parameters $\Delta c_{i t}^{\text {on }}$ associated with the "on" commitment status of generator $i$ at $t(£ / h)$

$\Delta \boldsymbol{c}_{\boldsymbol{i}}^{\text {off }} \quad$ Vector of uplift parameters $\Delta c_{i t}^{\text {off }}$ associated with the "off" commitment status of generator $i$ at $t(\mathfrak{f} / \mathrm{h})$

$\Delta \boldsymbol{\alpha}_{j}^{\boldsymbol{d}} \quad$ Vector of uplift parameters $\Delta \alpha_{j t}^{d}$ associated with the power demand of FD $j$ at $t(£ / M W h)$

$\boldsymbol{\Delta} \boldsymbol{\beta}_{\boldsymbol{j}}^{\boldsymbol{d}} \quad$ Vector of uplift parameters $\Delta \beta_{j t}^{d}$ associated with the square of the power demand of FD $j$ at $t\left(£ / \mathrm{MW}^{2} \mathrm{~h}\right)$

$\Delta \gamma_{j}^{d} \quad$ Uplift parameter associated with forgoing the activity of FD $j(\mathfrak{E})$

\section{Parameters}

D Vector of total inflexible demands $D_{t}(\mathrm{MW})$

$d_{j t}^{\min } \quad$ Minimum power limit of FD $j$ at $t(\mathrm{MW})$

$d_{j t}^{\max } \quad$ Maximum power limit of FD $j$ at $t(\mathrm{MW})$

$E_{j} \quad$ Energy requirement of the activity of FD $j$ (MWh)

D. Functions

$C_{i} \quad$ Cost function of generator $i$

$B_{j} \quad$ Benefit function of FD $j$

pro $_{i} \quad$ Profit function of generator $i$

$u t i_{j} \quad$ Utility function of FD $j$

$U_{i}^{g} \quad$ Uplift function of generator $i$

$U_{j}^{d} \quad$ Uplift function of FD $j$ 


\section{INTRODUCTION}

Uniform marginal prices cannot generally support competitive equilibrium solutions in markets with nonconvexities [1]-[7]. In other words, market participants' surplus maximizing self-schedule given the marginal prices determined by the centralized market clearing problem, is not generally consistent with the schedule calculated by the latter. In cases of such inconsistencies, the centralized schedules yield lower surpluses than self-scheduling, with this difference termed as surplus loss and the related effect as surplus sub-optimality. This effect is not acceptable on the basis of allowing all market participants to determine independently their production / consumption given the prices, and not by central intervention.

Previous work has identified non-convexities associated with the generation side of electricity markets, including binary (on/off) commitment decisions, fixed and start-up/shut-down costs, minimum stable generation constraints, and minimum up/down times [2]-[7]. As demonstrated in this literature, these non-convexities lead to generators' schedules inconsistency and profit sub-optimality effects.

Furthermore, different market schemes have been proposed to address such effects and achieve competitive equilibrium at the optimal solution of the centralized problem. After solving the initial mixed-integer centralized problem, authors in [2] solve a continuous version of the latter, with the binary commitment variables set equal to their optimal values. The dual variables of these equality constraints yield differentiated prices for the generators' commitment, which along with the uniform energy prices support an equilibrium solution. In [3][4], generators experiencing profit sub-optimality receive uplift payments that compensate their profit loss, and the electricity prices are optimized to minimize the total uplift payments.

However, approaches in [2]-[4] charge the total compensation of profit loss to the (completely inflexible in these works) demand side of the market, which is thus treated inequitably. To this end, authors in [5]-[7] propose the use of generalized uplift functions, which constitute additional revenues or payments for the generators. Due to the above shortcomings of uniform marginal prices, these functions include generator-specific linear and non-linear terms. The parameters of these functions along with the electricity prices are adjusted to achieve consistency for every generator and an equitable distribution of generators' profit loss compensation among the market participants. Considering a single period problem, the existence of such differentiated prices achieving consistency is proved in [5], while their calculation through an optimization problem is outlined in [6]. Since the approach followed in [6] is impractical in multi-period problems accounting for generators' time-coupling characteristics, an iterative cutting-plane algorithm for the calculation of uplift parameters and electricity prices is proposed in [7].

The introduction of flexible demand (FD) in power systems has attained continuously increasing interest due to its significant economic, technical, and environmental benefits [8]. In the deregulated environment, the realization of this potential is coupled with the integration of FD in electricity markets [9]. Authors in [10]-[12] have proposed different market clearing mechanisms considering FD participation and demonstrated the impact of FD on the market. However, previous work has not explored inconsistency and surplus suboptimality effects from the perspective of FD.

This paper identifies non-convexities associated with the operation of FD and demonstrates their relation with inconsistency and surplus sub-optimality effects. The generalized uplift approach and the iterative uplift computation algorithm of [5]-[7] are extended to support competitive equilibrium solutions under both generation and FD participation. Generalized uplift functions for FD participants are defined, accounting for both demand forgoing and rescheduling potentials, and a new rule is proposed for the equitable distribution of the total generators' profit loss and FD utility loss compensation among the market participants. These contributions are supported by case studies on a market with a day-ahead horizon and hourly resolution.

The rest of this paper is organized as follows. Section III derives operational models of FD and formulates the centralized market clearing problem under FD participation. Section IV identifies FD non-convexities and demonstrates the resulting inconsistency and surplus sub-optimality effects through simple examples. Section $\mathrm{V}$ details the extension of the generalized uplift approach and the cutting-plane uplift computation algorithm considering both generation and FD participation. Section VI presents the examined case study and illustrative results. Finally, Section VII concludes this work.

\section{Centralized Market Clearing under FleXIBLE DEMAND PARTICIPATION}

Based on participants' bids and offers, the market operator solves the social welfare maximization problem (1)-(2) to determine the clearing schedules $\boldsymbol{\xi}_{\boldsymbol{i}}^{*} \equiv\left[\boldsymbol{g}_{\boldsymbol{i}}^{*}, \boldsymbol{u}_{\boldsymbol{i}}^{*}\right] ; \forall i \in$ $I$ and $\boldsymbol{\psi}_{j}^{*} \equiv\left[\boldsymbol{d}_{\boldsymbol{j}}^{*}, v_{j}^{*}, \boldsymbol{w}_{j}^{*}\right] ; \forall j \in J$. A continuous version of (1)(2), with the binary variables set equal to their optimal values, is solved next to determine the electricity prices $\lambda^{*}$ (values of dual variables associated with constraints (2)).

$$
\max _{\substack{\xi_{i} \in \mathcal{G}_{i} ; \forall i \in I \\ \boldsymbol{\psi}_{j} \in \mathcal{D}_{j} ; \forall j \in J}} \sum_{j \in J} B_{j}\left(\boldsymbol{\psi}_{j}\right)-\sum_{i \in I} C_{i}\left(\xi_{i}\right)
$$

subject to: $\sum_{i \in I} g_{i t}=D_{t}+\sum_{j \in J} d_{j t} ; \forall t \in T$

In this paper, the generators' cost functions include variable, fixed, start-up and shut-down costs, and their operating constraints' sets include minimum stable and maximum generation limits, ramp rates, minimum-up and minimum-down times. The analytical formulation of these cost functions and constraints' sets follows the model presented in [13] and is not presented here for brevity reasons.

Regarding FD participants, two different demand flexibility potentials are considered in this paper. The first is associated with the ability to completely forgo a demand activity and the second is associated with the ability to reschedule the energy requirements of an activity in time. Without loss of generality, it is assumed that each FD participant carries out at most one activity over the market horizon. In order to account for the potential to forgo an activity, the benefit function of $\mathrm{FD} j$ is expressed by (3); the consumers receive zero benefit if the activity is forgone, or a 
constant benefit $B_{j}^{0}$ otherwise.

$B_{j}\left(\boldsymbol{\psi}_{j}\right)=B_{j}^{0} * v_{j}$

The operating constraints set $\mathcal{D}_{j}$ of a $\mathrm{FD} j$ includes the following constraints:

1. The total energy consumption is zero if the related activity is forgone, or equal to the fixed energy requirement of the activity otherwise.

$\sum_{t \in T} d_{j t} * 1 h=v_{j} * E_{j}$

2. When the FD is active (i.e. its demand is not zero), its power demand is bounded by a minimum and a maximum power limit.

$w_{j t} * d_{j t}^{\min } \leq d_{j t} \leq w_{j t} * d_{j t}^{\max } ; \forall t \in T$

\section{FleXible Demand Non-ConveXities AND IMPaCt ON CONSISTENCY AND SURPLUS OPTIMALITY}

In a competitive market, market participants act as selfinterested surplus-maximizing entities, given the electricity prices and subject to their operating constraints. These selfscheduling optimization problems for a generator $i$ and a FD $j$ are expressed by (6) and (7), and the resulting optimal schedules by $\xi_{i}^{s}$ and $\boldsymbol{\psi}_{j}^{s}$ respectively.

$\max _{\xi_{i} \in \mathcal{G}_{i}}\left(\right.$ pro $\left._{i} \equiv(\boldsymbol{\lambda})^{\prime} \boldsymbol{g}_{\boldsymbol{i}}-C_{i}\left(\xi_{\boldsymbol{i}}\right)\right)$

$\max _{\boldsymbol{\psi}_{j} \in \mathcal{D}_{j}}\left(u t i_{j} \equiv B_{j}\left(\boldsymbol{\psi}_{\boldsymbol{j}}\right)-(\boldsymbol{\lambda})^{\prime} \boldsymbol{d}_{\boldsymbol{j}}\right)$

According to Section II, $\xi_{i}^{s}$ and $\boldsymbol{\psi}_{j}^{s}$ given $\lambda^{*}$ are not generally consistent with $\xi_{i}^{*}$ and $\boldsymbol{\psi}_{j}^{*}$ respectively. In cases of such inconsistencies, $\xi_{i}^{*}$ and $\boldsymbol{\psi}_{j}^{*}$ generally entail lower surpluses than $\xi_{i}^{s}$ and $\boldsymbol{\psi}_{j}^{s}$ respectively, with this difference termed as surplus loss. The surplus loss for a generator $i$, a FD $j$ and the total surplus loss are respectively expressed by:

$$
\begin{aligned}
& \Delta \operatorname{loss}_{i}^{g} \equiv \operatorname{pro}_{i}\left(\lambda^{*}, \xi_{i}^{s}\right)-\operatorname{pro}_{i}\left(\lambda^{*}, \xi_{i}^{*}\right) \\
& \Delta \operatorname{loss}_{j}^{d} \equiv u t i_{j}\left(\lambda^{*}, \boldsymbol{\psi}_{j}^{s}\right)-u t i_{j}\left(\lambda^{*}, \boldsymbol{\psi}_{j}^{*}\right) \\
& \text { TotalLoss }=\sum_{i \in I} \Delta \operatorname{loss}_{i}^{g}+\sum_{j \in J} \Delta \operatorname{loss}_{j}^{d}
\end{aligned}
$$

Previous work has identified non-convexities associated with the generation side, yielding inconsistency and surplus sub-optimality effects (Section II). In this section, FD participants are examined from the same perspective. The first FD non-convexity is associated with the potential to forgo a demand activity and is mathematically captured by the binary decision variables $v_{j}$. A FD will choose to forgo its activity if its total utility is negative i.e. the total electricity payments incurred by the activity are higher than the benefit $B_{j}^{0}$ it derives from the activity, or carry out the activity if its total utility is positive.

The effect of this non-convexity is demonstrated through a single-period problem, where the market includes a) a FD $j$ with $E_{j}=12 \mathrm{MW} d_{j 1}^{\min }=5 \mathrm{MW}, d_{j 1}^{\max }=15 \mathrm{MW}$ and $B_{j}^{0}=150 £$ and b) a generator $i$ with a cost function $C_{i}\left(g_{i t}\right)=g_{i t}^{2}$ and without any non-convex characteristics.
Under centralized market clearing, the FD activity is carried out since $B_{j}^{0}$ is higher than the generation cost incurred to supply this demand (144£), and thus the optimal solution involves $g_{i t}^{*}=d_{j t}^{*}=12 \mathrm{MW}$ and $\lambda_{t}^{*}=24 \mathrm{f} / \mathrm{MWh}$. However, given this price, the FD would choose to forgo its activity, since its payments $\left(\lambda_{t}^{*} * d_{j t}^{*}=288 £\right)$ are higher than its benefit $B_{j}^{0}$; therefore, under the centralized solution, the FD incurs a utility loss of $138 £$.

Even if the potential to forgo a demand activity is neglected, FDs still exhibit a non-convex operating constraints' set, since their demand can take the values $d_{j t}=0$ and $d_{j t}=d_{j t}^{\min }$ but not any value in the range $\left(0, d_{j t}^{\text {min }}\right)$. A two-period problem is considered here, where the market includes a) a FD $j$ with $E_{j}=12 \mathrm{MWh}, d_{j 1}^{\min }=d_{j 2}^{\min }=$ $5 \mathrm{MW}$ and $\left.d_{j 1}^{\max }=d_{j 2}^{\max }=15 \mathrm{MW}, \mathrm{b}\right)$ inflexible demands with $D_{1}=10 \mathrm{MW}$ and $D_{2}=20 \mathrm{MW}$ and c) the same generator $i$ with the previous example. Under centralized market clearing, the FD activity is scheduled entirely at $t=1$ in order to flatten as much as possible the total demand profile and minimize the total generation costs, and the optimal solution involves $d_{j 1}^{*}=12 \quad \mathrm{MW}, \quad d_{j 2}^{*}=0 \mathrm{MW}, \lambda_{1}^{*}=$ $44 £ / \mathrm{MWh}$ and $\lambda_{2}^{*}=40 £ / \mathrm{MWh}$. However, given these prices the FD would choose to be scheduled entirely at $t=2$ since this period exhibits a lower price; therefore, under the centralized solution, the FD incurs a utility loss of $48 £$.

If the same problem is considered with $d_{j 1}^{\min }=d_{j 2}^{\text {min }}=0$, the optimal centralized solution flattens completely the total demand profile with $d_{j 1}^{*}=11 \mathrm{MW}, d_{j 2}^{*}=1 \mathrm{MW}$ and $\lambda_{1}^{*}=$ $\lambda_{2}^{*}=42 \mathfrak{f} / \mathrm{MWh}$. Given these prices, any feasible solution of the FD's self-scheduling problem including its above centralized schedule is an optimal one, and therefore the FD does not incur utility loss. It can be thus concluded that FDs' non-convexities associated with their ability to forgo activities and minimum power levels yield schedules' inconsistency and utility sub-optimality effects.

\section{Generalized Uplifts Under FleXiBle Demand PARTICIPATION}

\section{A. Formulation of Minimum Generalized Uplift Problem}

A generalized uplift approach is proposed in [5]-[7] to eliminate schedules' inconsistency and profit sub-optimality caused by generation non-convexities, and achieve competitive equilibrium at the centralized solution. The generalized uplift function $U_{i}^{g}(11)$ constitutes additional revenue $(U>0)$ or payment $(U<0)$ for generator $i$, and includes a set of generator-specific parameters $\Delta \pi_{i}^{g}=$ $\left[\Delta \boldsymbol{\alpha}_{i}^{g}, \boldsymbol{\Delta} \boldsymbol{c}_{i}^{\text {on }}, \boldsymbol{\Delta} \boldsymbol{c}_{\boldsymbol{i}}^{\boldsymbol{o f f}}\right]$ associated with the power output, the "on" commitment status and the "off" commitment status of generator $i$ respectively. These parameters are adjusted so that generators' new or augmented self-scheduling is consistent with the centralized solution. Along with these parameters, the new electricity prices $\lambda^{N}$ faced by the (inflexible) demand are adjusted to ensure that the latter contributes at a desired level in the compensation of generators' profit loss. This adjustment is carried out through an optimization problem, denoted as the minimum generalized uplift problem [7]. 
$U_{i}^{g}\left(\xi_{i}, \Delta \boldsymbol{\pi}_{i}^{g}\right)=\sum_{t \in T}\left[\Delta \alpha_{i t}^{g} g_{i t}+\Delta c_{i t}^{o n} u_{i t}+\Delta c_{i t}^{o f f}\left(1-u_{i t}\right)\right](11)$

In the same vein, we propose a generalized uplift function $U_{j}^{d}$ (12) applying to FD $j$. It includes a set of adjustable FDspecific parameters $\Delta \boldsymbol{\pi}_{j}^{\boldsymbol{d}}=\left[\boldsymbol{\Delta} \boldsymbol{\alpha}_{j}^{\boldsymbol{d}}, \boldsymbol{\Delta} \boldsymbol{\beta}_{j}^{\boldsymbol{d}}, \Delta \gamma_{j}^{d}\right]$ associated with the power input, the square of the power input and forgoing the activity of FD $j$ respectively. As demonstrated in Section VI, the proposed uplift functions provide adequate degrees of freedom to achieve consistency for all FDs in the market.

$$
U_{j}^{d}\left(\boldsymbol{\psi}_{j}, \Delta \boldsymbol{\pi}_{j}^{d}\right)=\Delta \gamma_{j}^{d}\left(1-v_{j}\right)+\sum_{t \in T}\left[\Delta \alpha_{j t}^{d} d_{j t}+\Delta \beta_{j t}^{d}\left(d_{j t}\right)^{2}\right]
$$

Given the above uplift functions, the augmented selfscheduling optimization problems involve the maximization of the augmented profit of generator $i$ (13) and the augmented utility of FD $j$ (14).

$$
\begin{aligned}
& \operatorname{pro}_{i}\left(\lambda^{N}, \xi_{i}, \Delta \boldsymbol{\pi}_{i}^{g}\right) \equiv\left(\lambda^{N}\right)^{\prime} \boldsymbol{g}_{\boldsymbol{i}}-C_{i}\left(\xi_{i}\right)+U_{i}^{g}\left(\xi_{i}, \Delta \boldsymbol{\pi}_{\boldsymbol{i}}^{\boldsymbol{g}}\right) \\
& u_{i}\left(\boldsymbol{\lambda}^{N}, \boldsymbol{\psi}_{\boldsymbol{j}}, \Delta \boldsymbol{\pi}_{\boldsymbol{j}}^{\boldsymbol{d}}\right) \equiv B_{j}\left(\boldsymbol{\psi}_{\boldsymbol{j}}\right)-\left(\boldsymbol{\lambda}^{N}\right)^{\prime} \boldsymbol{d}_{\boldsymbol{j}}+U_{j}^{d}\left(\boldsymbol{\psi}_{\boldsymbol{j}}, \Delta \boldsymbol{\pi}_{\boldsymbol{j}}^{\boldsymbol{d}}\right)
\end{aligned}
$$

The minimum generalized uplift problem includes the following constraints:

1) The solution of the centralized market clearing problem is identical to the solution of the augmented self-scheduling problems for all market participants (15)-(16).

$$
\begin{gathered}
\xi_{i}^{*}=\xi_{i}^{a} \equiv \arg \max _{\xi_{i} \in \mathcal{G}_{i}} \operatorname{pro}_{i}\left(\lambda^{N}, \xi_{i}, \Delta \boldsymbol{\pi}_{\boldsymbol{i}}^{\boldsymbol{g}}\right) ; \forall i \in I \\
\boldsymbol{\psi}_{j}^{*}=\boldsymbol{\psi}_{j}^{\boldsymbol{a}} \equiv \arg \max _{\boldsymbol{\psi}_{j} \in \mathcal{D}_{j}} u t i_{j}\left(\boldsymbol{\lambda}^{\boldsymbol{N}}, \boldsymbol{\psi}_{\boldsymbol{j}}, \Delta \boldsymbol{\pi}_{\boldsymbol{j}}^{\boldsymbol{d}}\right) ; \forall j \in J
\end{gathered}
$$

2) Conservation of monetary flow within the market should be satisfied, meaning that the total revenues collected by the consumers are equal to the total payments paid to the generators, including the uplifts (17). The combination of (17) and (2) implies that the sum of uplifts should be zero (18).

$\sum_{j \in J}\left[\left(\lambda^{N}\right)^{\prime} \boldsymbol{d}_{j}^{*}-U_{j}^{d}\left(\boldsymbol{\psi}_{j}^{*}, \Delta \boldsymbol{\pi}_{j}^{d}\right)\right]+\left(\lambda^{N}\right)^{\prime} \boldsymbol{D}=\sum_{i \in I}\left[\left(\lambda^{N}\right)^{\prime} \boldsymbol{g}_{i}^{*}+\right.$ $\left.U_{i}^{g}\left(\xi_{i}^{*}, \Delta \pi_{i}^{g}\right)\right]$

$\sum_{i \in I} U_{i}^{g}\left(\xi_{i}^{*}, \Delta \boldsymbol{\pi}_{i}^{g}\right)+\sum_{j \in J} U_{j}^{d}\left(\boldsymbol{\psi}_{j}^{*}, \Delta \boldsymbol{\pi}_{j}^{\boldsymbol{d}}\right)=0$

3) The difference between a participant's surplus under augmented self-scheduling and self-scheduling without uplifts determines the contribution of this participant to the compensation of the total surplus loss [6]. This contribution is expressed by (19), (20), and (21) for generator $i, \operatorname{FD} j$, and the inflexible demand respectively.

$\Delta \operatorname{cont}_{i}^{g}\left(\lambda^{N}, \Delta \boldsymbol{\pi}_{i}^{g}\right) \equiv \operatorname{pro}_{i}\left(\lambda^{*}, \xi_{i}^{s}, \mathbf{0}\right)-\operatorname{pro}_{i}\left(\lambda^{N}, \xi_{i}^{a}, \Delta \pi_{i}^{g}\right) \geq 0$

$\Delta \operatorname{cont}_{j}^{d}\left(\lambda^{N}, \Delta \boldsymbol{\pi}_{j}^{d}\right) \equiv u t i_{j}\left(\lambda^{*}, \boldsymbol{\psi}_{j}^{s}, \mathbf{0}\right)-u t i_{j}\left(\lambda^{N}, \boldsymbol{\psi}_{j}^{\boldsymbol{a}}, \Delta \boldsymbol{\pi}_{j}^{\boldsymbol{d}}\right) \geq 0$

$\Delta \operatorname{cont}^{\text {inf }}\left(\boldsymbol{\lambda}^{N}\right) \equiv\left(\lambda^{N}\right)^{\prime} \boldsymbol{D}-\left(\boldsymbol{\lambda}^{*}\right)^{\prime} \boldsymbol{D} \geq 0$

The combination of (2), (8)-(10), (15)-(16) and (19)-(21) yields (22), which expresses that the total surplus loss is equal to the total compensation contribution by all participants.
TotalLoss $=\Delta \operatorname{cont}^{\text {inf }}\left(\lambda^{N}\right)+\sum_{i \in I} \Delta \operatorname{cont}_{i}^{g}\left(\lambda^{N}, \Delta \pi_{i}^{g}\right)+$ $\sum_{j \in J} \Delta \operatorname{cont}_{j}^{d}\left(\lambda^{N}, \Delta \boldsymbol{\pi}_{j}^{d}\right)$

Authors in [6] proposed two market rules to achieve an equitable distribution of the compensation for the total surplus loss among the market participants: a) the total compensation is divided equally among generators and inflexible demand and b) the ratio between profit under augmented selfscheduling and self-scheduling without uplifts is set equal for all generators. In the context of this paper, the authors believe that an equitable compensation distribution between generators, FDs and inflexible demand cannot be determined unambiguously. However, an extended version of the latter market rule is proposed, where the ratio $R(0<R<1)$ between surplus under augmented self-scheduling and self-scheduling without uplifts is set equal for all generators, FDs and inflexible demand (23)-(25).

$$
\begin{aligned}
& \operatorname{pro}_{i}\left(\lambda^{N}, \xi_{i}^{\boldsymbol{a}}, \Delta \boldsymbol{\pi}_{\boldsymbol{i}}^{\boldsymbol{g}}\right)=\operatorname{pro}_{i}\left(\boldsymbol{\lambda}^{*}, \xi_{i}^{s}, \mathbf{0}\right) * R ; \forall i \in I \\
& u t i_{j}\left(\lambda^{N}, \boldsymbol{\psi}_{j}^{\boldsymbol{a}}, \Delta \boldsymbol{\pi}_{j}^{\boldsymbol{d}}\right)=u t i_{j}\left(\boldsymbol{\lambda}^{*}, \boldsymbol{\psi}_{\boldsymbol{j}}^{\boldsymbol{s}}, \mathbf{0}\right) * R ; \forall j \in J \\
& \left(\boldsymbol{\lambda}^{*}\right)^{\prime} \boldsymbol{D}=\left(\boldsymbol{\lambda}^{N}\right)^{\prime} \boldsymbol{D} * R
\end{aligned}
$$

Substituting (23) - (25) into (22) yields:

$$
\begin{aligned}
& \overbrace{\text { TotalLoss }}^{A}= \\
& (1-R) * \overbrace{\left[\sum_{i \in I} \operatorname{pro}_{i}\left(\lambda^{*}, \xi_{i}^{s}, \mathbf{0}\right)+\sum_{j \in J} u t i_{j}\left(\lambda^{*}, \boldsymbol{\psi}_{j}^{\boldsymbol{s}}, \mathbf{0}\right)\right]}^{B}+ \\
& \left(\frac{1}{R}-1\right) * \underbrace{\left(\lambda^{*}\right)^{\prime} \boldsymbol{D}}_{C}
\end{aligned}
$$

Along with the condition $0<R<1$, $\mathrm{R}$ is calculated as:

$R=\frac{-(A+C-B)+\sqrt{(A+C-B)^{2}+4 B C}}{2 B}$

Equation (25), along with the assumption that the electricity price is uniformly increased across all periods of the market horizon [6], fixes the new electricity prices according to $(28)$.

$\lambda_{t}^{N}=\lambda_{t}^{*}+\frac{\left(\frac{1}{R}-1\right) \sum_{t \in T} \lambda_{t}^{*} D_{t}}{\sum_{t \in T} D_{t}} ; \forall t \in T$

As in [6], the objective function of the minimum generalized uplift problem involves the minimization of the square norm of uplift parameters (29). The selection of this objective function aims to introduce the minimum discrimination by the employment of uplifts.

$$
\min _{\substack{\Delta \pi_{i}^{\boldsymbol{g}} ; \forall i \in I \\ \Delta \boldsymbol{\pi}_{j}^{\boldsymbol{d}} ; \forall j \in J}} \sum_{i \in I}\left\|\Delta \boldsymbol{\pi}_{\boldsymbol{i}}^{\boldsymbol{g}}\right\|^{2}+\sum_{j \in J}\left\|\Delta \boldsymbol{\pi}_{\boldsymbol{j}}^{\boldsymbol{d}}\right\|^{2}
$$

\section{B. Iterative Computation of Uplift Parameters}

In order to solve the minimum generalized uplift problem, the optimal solutions $\xi_{i}^{a} ; \forall i \in I$ and $\boldsymbol{\psi}_{j}^{\boldsymbol{a}} ; \forall j \in J$ of the augmented self-scheduling problems need to be analytically expressed in terms of the uplift parameters and new electricity prices, so as to enforce the equal schedule conditions (15)(16). As discussed in [7], such analytical derivations are impractical for multi-period market problems accounting for 
participants' time-coupling properties. In order to address this challenge, authors in [7] proposed an iterative cutting-plane algorithm for the solution of the minimum generalized uplift problem, and proved its convergence and optimality. This algorithm iteratively restricts the feasible set of uplift parameters and new electricity prices, through the sequential generation of profit cutting planes, to impose indirectly conditions (15)-(16). In this section, this algorithm is extended to compute the uplift parameters of both generation and FD participants and includes the following steps:

(Step 1) The centralized market clearing problem (1)-(2) is solved to obtain $\boldsymbol{\xi}_{i}^{*} ; \forall i \in I, \boldsymbol{\psi}_{j}^{*} ; \forall j \in J$ and $\boldsymbol{\lambda}^{*}$. The iteration counter is set to zero $r=0$, as well as the initial values of the uplift parameters $\left(\Delta \boldsymbol{\pi}_{\boldsymbol{i}}^{g}\right)^{[\mathbf{0}]}=\mathbf{0} ; \forall i \in I$ and $\left(\Delta \boldsymbol{\pi}_{j}^{\boldsymbol{d}}\right)^{[\mathbf{0}]}=$ $\mathbf{0} ; \forall j \in J$. The sets of generators' profit and FDs' utility cutting planes are initialized to the entire space of uplift parameters $\Xi_{i}^{[0]}=\mathbb{R}^{3 T} ; \forall i \in I$ and $\Psi_{j}^{[0]}=\mathbb{R}^{2 T+1} ; \forall j \in J$. In this paper, the new electricity prices $\lambda^{N}$ are fixed by the equitable compensation distribution rule (28), and therefore constitute fixed inputs to the remaining steps of the algorithm.

(Step 2) The augmented self-schedule of each market participant given the latest value of the uplift parameters and the new electricity prices, is calculated:

$$
\begin{aligned}
& \left(\xi_{i}^{\boldsymbol{a}}\right)^{[r+1]}=\arg \max _{\xi_{i} \in \mathcal{G}_{i}} \operatorname{pro}_{i}\left(\lambda^{N}, \xi_{\boldsymbol{i}},\left(\Delta \boldsymbol{\pi}_{\boldsymbol{i}}^{\boldsymbol{g}}\right)^{[r]}\right) ; \forall i \in I \\
& \left(\boldsymbol{\psi}_{j}^{\boldsymbol{a}}\right)^{[r+1]}=\arg \max _{\boldsymbol{\psi}_{j} \in \mathcal{D}_{j}} u t i_{j}\left(\lambda^{N}, \boldsymbol{\psi}_{j},\left(\Delta \boldsymbol{\pi}_{j}^{\boldsymbol{d}}\right)^{[r]}\right) ; \forall j \in J
\end{aligned}
$$

(Step 3) If the augmented self-schedule of a generator $i$ or a FD $j$ obtained in (Step 2) is different than the respective centralized schedule, a new cut is added to the respective cutting plane set (34)-(35). In mathematical terms, if:

$$
\begin{aligned}
& \left(\xi_{i}^{\boldsymbol{a}}\right)^{[r+1]} \neq \boldsymbol{\xi}_{i}^{*} \\
& \left(\boldsymbol{\psi}_{j}^{\boldsymbol{a}}\right)^{[r+1]} \neq \boldsymbol{\psi}_{j}^{*}
\end{aligned}
$$

then respectively (where $\epsilon^{g}$ and $\epsilon^{d}$ are strictly positive parameters [7]):

$\Xi_{i}^{[r+1]}=\Xi_{i}^{[r]} \cap\left\{\Delta \pi_{i}^{g} \in \mathbb{R}^{3 T}: \operatorname{pro}_{i}\left(\lambda^{N},\left(\xi_{i}^{a}\right)^{[r+1]}, \Delta \pi_{i}^{g}\right) \leq\right.$

$\left.\operatorname{pro}_{i}\left(\lambda^{N}, \boldsymbol{\xi}_{i}^{*}, \Delta \boldsymbol{\pi}_{i}^{g}\right)-\epsilon^{g}\right\}$

$\Psi_{j}^{[r+1]}=\Psi_{j}^{[r]} \cap\left\{\Delta \boldsymbol{\pi}_{j}^{\boldsymbol{d}} \in \mathbb{R}^{2 T+1}: u t i_{j}\left(\lambda^{N},\left(\boldsymbol{\psi}_{j}^{\boldsymbol{a}}\right)^{[r+1]}, \Delta \boldsymbol{\pi}_{j}^{\boldsymbol{d}}\right) \leq\right.$

$\left.u t i_{j}\left(\lambda^{N}, \boldsymbol{\psi}_{j}^{*}, \Delta \boldsymbol{\pi}_{j}^{d}\right)-\epsilon^{d}\right\}$

else respectively:

$\Xi_{i}^{[r+1]}=\Xi_{i}^{[r]}$

$\Psi_{j}^{[r+1]}=\Psi_{j}^{[r]}$

(Step 4) If conditions (36)-(37) are satisfied for every participant, the algorithm terminates. If not, a new set of trial uplift parameters $\left(\Delta \boldsymbol{\pi}_{i}^{g}\right)^{[\boldsymbol{r}+\mathbf{1}]}$ and $\left(\Delta \boldsymbol{\pi}_{j}^{\boldsymbol{d}}\right)^{[\boldsymbol{r}+\mathbf{1}]}$ is calculated through the solution of problem (29) subject to the monetary flow conservation constraint (18), the equitable compensation distribution constraints (23)-(24) and (28), the constraints of the cutting plane sets (38)-(39), and constraints (40) ensuring that the objective function of FDs' augmented self-scheduling problems (31) is bounded. The iteration counter is then incremented by 1 and the algorithm returns to (Step 2).

$\Delta \boldsymbol{\pi}_{\boldsymbol{i}}^{\boldsymbol{g}} \in \Xi_{i}^{[r+1]} ; \forall i \in I$

$\Delta \boldsymbol{\pi}_{j}^{\boldsymbol{d}} \in \Psi_{j}^{[r+1]} ; \forall j \in J$

$\Delta \beta_{j t}^{d}<0 ; \forall j \in J, \forall t \in T$

\section{CASE STUdy}

The examined case study involves the application of the proposed generalized uplift approach to a market with a daily horizon and hourly resolution, using the optimization software FICO $^{\mathrm{TM}}$ Xpress v7.7 [14]. The market includes 7 generation participants, whose fixed costs $a_{i}$, linear $b_{i}$ and quadratic $c_{i}$ parameters of their variable cost functions, start-up $C_{i}^{u}$ and shut-down $C_{i}^{d}$ costs, minimum stable $g_{i}^{\text {min }}$ and maximum $g_{i}^{\max }$ generation limits, ramp rates $R R_{i}$ (identical rates assumed for ramp-up and ramp-down), minimum-up $U T_{i}$ and minimum-down $D T_{i}$ times, and initial commitment status $u_{i 0}$ and output $g_{i 0}$ are given in Table I. At the beginning of $t=1$,

\begin{tabular}{|c|c|c|c|c|c|c|c|}
\hline Gen $i$ & 1 & 2 & 3 & 4 & 5 & 6 & 7 \\
\hline$a_{i}(\mathfrak{f} / \mathbf{h})$ & 18,431 & 17,005 & 13,755 & 9,930 & 9,900 & 8,570 & 7,530 \\
\hline$b_{i}(\mathfrak{f} / \mathbf{M W h})$ & 5.5 & 30 & 35 & 60 & 80 & 95 & 100 \\
\hline$c_{i}\left(\mathfrak{f} / \mathbf{M W}^{2} \mathbf{h}\right)$ & 0.0002 & 0.0007 & 0.0010 & \begin{tabular}{|l|}
0.0064 \\
\end{tabular} & 0.0070 & 0.0082 & 0.0098 \\
\hline$C_{i}^{u}(\mathfrak{f})$ & $4,000,000$ & 325,000 & 142,500 & 72,000 & 55,000 & 31,000 & 11,200 \\
\hline 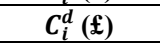 & 800,000 & 28,500 & 18,500 & 14,400 & 12,000 & 10,000 & 8,400 \\
\hline$g_{i}^{\min }(\mathrm{MW})$ & 3,292 & 2,880 & 1,512 & 667 & 650 & 288 & 275 \\
\hline$g_{i}^{\max }$ (MW) & 6,584 & 5,760 & 3,781 & 3,335 & 3,252 & 2,880 & 2,748 \\
\hline$R_{R}(\mathrm{MW} / \mathrm{h})$ & 1,317 & 1,152 & 1,512 & 1,334 & 1,951 & 1,728 & 2,198 \\
\hline$U T_{i}(\mathrm{~h})$ & 24 & 20 & 16 & 10 & 8 & 5 & 4 \\
\hline$D T_{i}(\mathrm{~h})$ & 24 & 20 & 16 & 10 & 8 & 5 & 4 \\
\hline$u_{i 0}$ & 1 & 1 & 1 & 1 & 1 & 0 & 0 \\
\hline$g_{i 0}(\mathrm{MW})$ & 5,070 & 4,435 & 2,912 & 2,568 & 2,504 & 0 & 0 \\
\hline
\end{tabular}
generators with $u_{i 0}=1$ and $u_{i 0}=0$ are assumed to be on and off for $U T_{i}$ and $D T_{i}$ hours respectively.

\section{TABLE I. GENERATION PARTICIPANTS’ CHARACTERISTICS}

Furthermore, the market includes 8 FD participants able to forgo and reschedule their demand activities, with parameters given in Table II. The minimum and maximum power limits of each FD are assumed identical at every hour of their scheduling period (denoted by $d_{j}^{\text {min }}$ and $d_{j}^{\text {max }}$ respectively) and zero at the rest of the hours. FD participants 1-4 can be scheduled during night/morning hours, representing domestic FD, and FD participants 5-8 during midday hours, representing commercial/industrial FD.

Under centralized market clearing, domestic and commercial/industrial FDs fill the inflexible demand's night and midday valleys respectively, flattening significantly the total demand profile, as shown on Fig. 1. However, these valleys and subsequently the centralized prices at these periods are not completely flattened, due to the minimum power levels of FD participants. 
TABLE II. FD PARTICIPANTS' CHARACTERISTICS

\begin{tabular}{|c|c|c|c|c|}
\hline FD $j$ & 1 & 2 & 3 & 4 \\
\hline$B_{j}^{0}(£$ mil $)$ & 0.187 & 0.486 & 0.311 & 0.334 \\
\hline$E_{j}(\mathrm{MWh})$ & 2,677 & 3,241 & 2,071 & 2,228 \\
\hline$d_{j}^{\min }(\mathrm{MW})$ & 1,328 & 1,620 & 497 & 980 \\
\hline$d_{j}^{\max }(\mathrm{MW})$ & 2,142 & 2,593 & 1,657 & 1,782 \\
\hline Scheduling period (h) & $18-8$ & $18-7$ & $19-8$ & $19-9$ \\
\hline FD $j$ & 5 & 6 & 7 & 8 \\
\hline$B_{j}^{0}(£$ mil $)$ & 0.352 & 0.197 & 0.175 & 0.113 \\
\hline$E_{j}(\mathbf{M W h})$ & 3,119 & 1,315 & 1,166 & 958 \\
\hline$d_{j}^{\min }(\mathrm{MW})$ & 1,273 & 421 & 583 & 479 \\
\hline$d_{j}^{\max }(\mathrm{MW})$ & 2,495 & 1,052 & 932 & 767 \\
\hline Scheduling period (h) & $9-17$ & $10-17$ & $9-17$ & $11-18$ \\
\hline
\end{tabular}

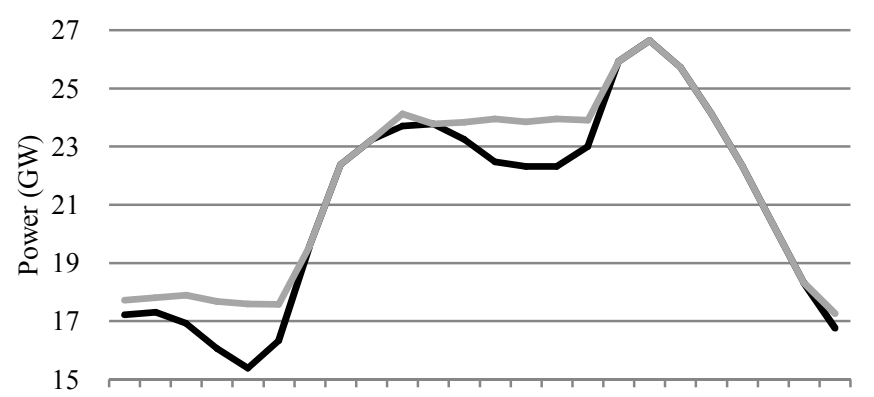

$\begin{array}{llllllllll}1 & 2 & 3 & 4 & 5 & 6 & 7 & 8 & 9 & 101112131415161718192021222324\end{array}$

— Inflex. Dem. Time(h), Total Dem.

Fig. 1. Inflexible demand and total demand under centralized market clearing.

As illustrated in Fig. 2, FD participants' self-scheduling given the centralized prices and no uplifts is not consistent with the centralized schedule. These inconsistencies are associated with both demand flexibility potentials. Considering the ability to reschedule their activities, under self-scheduling, FDs concentrate at the lowest-priced hours within their scheduling period, which is not consistent with the centralized solution involving an as-flat-as-possible total demand profile.

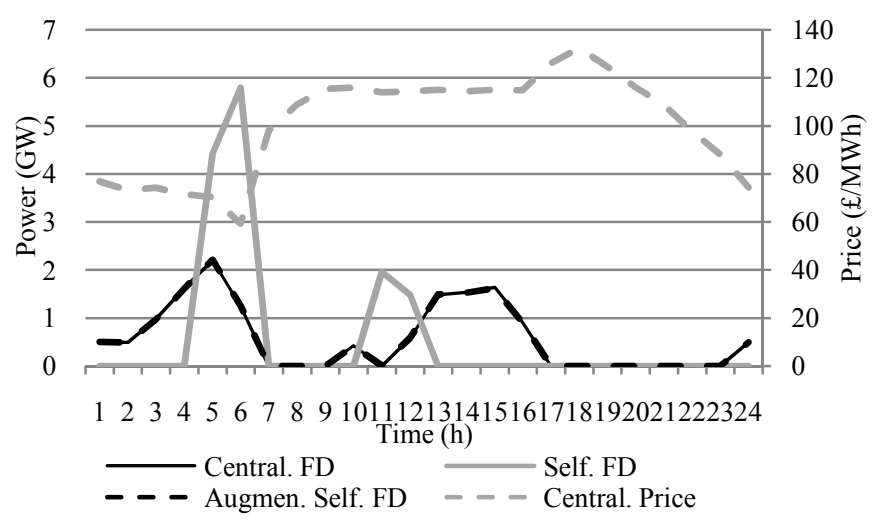

Fig. 2. Comparison of total FD under centralized market clearing, selfscheduling and augmented-self scheduling.

Considering the ability to forgo their demand activities, FD 5 would choose to do so under self-scheduling, since its benefit $B_{5}^{0}=£ 0.352 \mathrm{mil}$ is lower than the lowest payment it could achieve by optimally scheduling within its feasible scheduling period ( $£ 0.356 \mathrm{mil})$. On the other hand, under centralized clearing, its activity is not forgone since $B_{5}^{0}$ is higher than the extra generation cost incurred to supply the demand of this activity ( $£ 0.341 \mathrm{mil})$. The opposite case holds for FD 1. Under self-scheduling, the latter would choose to carry out its activity, since its benefit $B_{1}^{0}=£ 0.187 \mathrm{mil}$ is higher than the lowest payment it could achieve by optimally scheduling its activity ( $£ 0.174$ mil). Under centralized clearing however, its demand activity is forgone since $B_{1}^{0}$ is lower than the extra generation cost incurred to satisfy its demand (£0.199 mil).

As illustrated in Fig. 3, the generation side also exhibits inconsistencies between centralized scheduling and selfscheduling, associated with generation non-convexities explored in [2]-[7]. All the above inconsistencies are translated into surplus losses, demonstrated in Table III. In order to eliminate these inconsistency and surplus suboptimality effects, the proposed generalized uplift approach was applied. The parameter $R$ associated with the equitable distribution of the total surplus loss compensation was calculated as $R=99.91 \%$ according to (27). The new electricity prices are then calculated according to (28) as $\lambda_{t}^{N}=\lambda_{t}^{*}+0.098 £ ; \forall t \in T$.

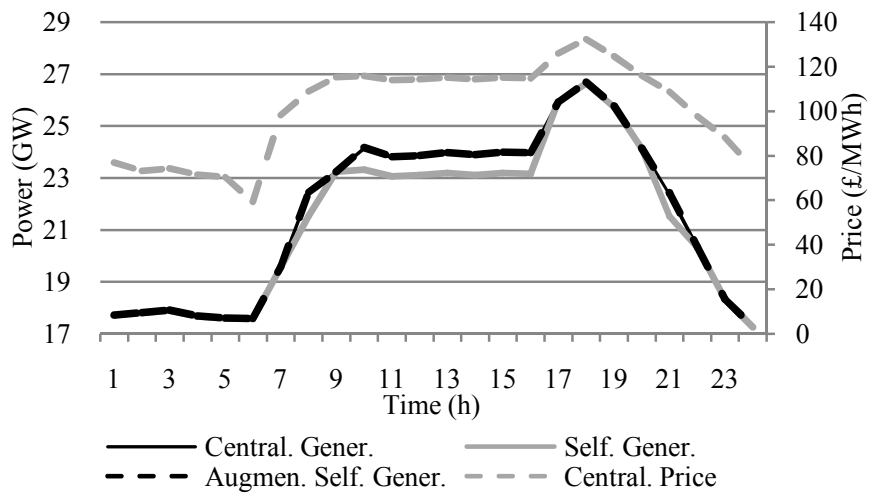

Fig. 3. Comparison of total generation under centralized market clearing, self-scheduling and augmented-self scheduling.

The uplift parameters' iterative computation algorithm was then applied with $\epsilon^{g}=\epsilon^{d}=0.01 £$ in (34)-(35), and converged after 57 iterations and 197 seconds of computational time on a computer, with a 6 -core, $3.47 \mathrm{GHz}$ Intel(R) Xeon(R) X5690 processor and 192GB of RAM. As illustrated in Fig. 2 and 3, FD and generation participants' augmented self-scheduling given the values of the uplift parameters calculated by the iterative algorithm and the new electricity prices, is consistent with the centralized schedule. As shown in Table III, the total compensation contribution by all participants exactly cancels out the total surplus loss.

Table IV presents the required types of uplift parameters (types without which the iterative algorithm cannot converge to a consistent augmented self-schedule) for the different FD participants. FD 1 requires only the uplift parameter $\Delta \gamma_{j}^{d}$, since its demand activity is forgone under centralized clearing and uplifts associated with its power demand cannot have any effect in achieving consistency. On the other hand, the rest of 
TABLE III

CASe Study Results

\begin{tabular}{|c|c|c|c|c|c|c|}
\hline \multicolumn{2}{|c|}{$\begin{array}{c}\text { Market } \\
\text { Participants }\end{array}$} & Centralized surplus $(\mathfrak{f})$ & Self-scheduling surplus $(\mathfrak{f})$ & $\begin{array}{c}\text { Augmented self- } \\
\text { scheduling surplus (£) }\end{array}$ & Surplus loss (£) & $\begin{array}{l}\text { Contribution to } \\
\text { surplus loss }(\mathfrak{f})\end{array}$ \\
\hline \multirow{7}{*}{ Generator $i$} & 1 & $14,420,186$ & $14,420,186$ & $14,406,600$ & 0 & 13,587 \\
\hline & 2 & $8,871,427$ & $8,871,427$ & $8,863,090$ & 0 & 8,337 \\
\hline & 3 & $5,400,546$ & $5,400,546$ & $5,395,470$ & 0 & 5,076 \\
\hline & 4 & $1,592,630$ & $1,592,630$ & $1,591,130$ & 0 & 1,500 \\
\hline & 5 & 467,944 & 467,944 & 467,505 & 0 & 439 \\
\hline & 6 & 73,702 & 77,087 & 77,015 & 3,385 & 72 \\
\hline & 7 & 13,977 & 20,415 & 20,396 & 6,438 & 19 \\
\hline \multirow{8}{*}{$\mathrm{FD} j$} & 1 & 0 & 13,806 & 13,793 & 13,806 & 13 \\
\hline & 2 & 256,123 & 275,834 & 275,575 & 19,712 & 259 \\
\hline & 3 & 158,256 & 182,246 & 182,074 & 23,990 & 172 \\
\hline & 4 & 187,218 & 191,067 & 190,888 & 3,849 & 179 \\
\hline & 5 & $-6,229$ & 0 & 0 & 6,229 & 0 \\
\hline & 6 & 46,008 & 47,183 & 47,139 & 1,175 & 44 \\
\hline & 7 & 41,532 & 41,763 & 41,723 & 231 & 39 \\
\hline & 8 & 3,268 & 3,672 & 3,668 & 404 & 3 \\
\hline \multicolumn{2}{|c|}{ Inflexible demand } & $52,629,406$ & $52,629,406$ & $52,678,920$ & 0 & 49,514 \\
\hline \multicolumn{2}{|c|}{ Total } & & & & 79,221 & 79,221 \\
\hline
\end{tabular}

the FDs do not require $\Delta \gamma_{j}^{d}$ since their activities are not forgone under centralized clearing, and $\Delta \gamma_{j}^{d}$ cannot have any effect in achieving consistency. For these FDs, uplifts parameters associated with the square of their demand are required to limit their tendency to concentrate at the lowestpriced periods.

TABLE IV. REQUIRED UPLIFT PARAMETERS OF FD PARTICIPANTS

\begin{tabular}{|c|c|}
\hline FD $\boldsymbol{j}$ & Required uplift parameters \\
\hline 1 & $\Delta \gamma_{j}^{d}$ \\
\hline 2 & $\boldsymbol{\Delta} \boldsymbol{\alpha}_{j}^{\boldsymbol{d}}$ and $\boldsymbol{\Delta} \boldsymbol{\beta}_{j}^{\boldsymbol{d}}$ \\
\hline 3 & $\boldsymbol{\Delta} \boldsymbol{\alpha}_{j}^{\boldsymbol{d}}$ and $\boldsymbol{\Delta} \boldsymbol{\beta}_{j}^{\boldsymbol{d}}$ \\
\hline 4 & $\boldsymbol{\Delta} \boldsymbol{\alpha}_{j}^{\boldsymbol{d}}$ and $\boldsymbol{\Delta} \boldsymbol{\beta}_{j}^{\boldsymbol{d}}$ \\
\hline 5 & $\boldsymbol{\Delta} \boldsymbol{\alpha}_{j}^{d}$ and $\boldsymbol{\Delta} \boldsymbol{\beta}_{j}^{d}$ \\
\hline 6 & $\boldsymbol{\Delta} \boldsymbol{\alpha}_{j}^{d}$ and $\boldsymbol{\Delta} \boldsymbol{\beta}_{j}^{\boldsymbol{d}}$ \\
\hline 7 & $\boldsymbol{\Delta} \boldsymbol{\alpha}_{j}^{d}$ and $\boldsymbol{\Delta} \boldsymbol{\beta}_{j}^{d}$ \\
\hline 8 & $\boldsymbol{\Delta} \boldsymbol{\alpha}_{j}^{d}$ and $\boldsymbol{\Delta} \boldsymbol{\beta}_{j}^{\boldsymbol{d}}$ \\
\hline
\end{tabular}

\section{CONCLUSIONS}

This paper explores the inability of uniform marginal prices in supporting competitive equilibrium solutions from the perspective of the FD side of electricity markets. Nonconvexities associated with the operation of FD are identified, including its ability to forgo load processes and minimum power levels. The relation of these non-convexities with schedules' inconsistency and surplus sub-optimality effects are demonstrated through simple one- and two-time period examples and a larger case study with a day-ahead horizon and hourly resolution.

The generalized uplift approach of [5]-[7] is extended to support competitive equilibrium solutions under both generation and FD participation by defining suitable generalized uplift functions for FD participants. These include quadratic pricing terms to limit the tendency of FD to concentrate at the lowest-priced periods and binary terms associated with their ability to forgo demand activities. Finally, a new rule is proposed for the equitable distribution of the total surplus loss among the market participants, setting the ratio between surpluses under self-scheduling with uplifts and without uplifts equal for all participants.

\section{REFERENCES}

[1] H. E. Scarf, "The allocation of resources in the presence of indivisibilities," J. Econ. Perspect., vol. 8, no. 4, pp. 111-128, Jan. 1994.

[2] R. P. O’Neill, P. M. Sotkiewicz, B. F. Hobbs, M. H. Rothkopf and W. R. Stewart Jr., "Efficient market-clearing prices in markets with nonconvexities," Eur. J. Oper. Res., vol. 164, no. 1 pp. 269-285, Jul. 2005.

[3] W. W. Hogan and B. J. Ring, "On minimum-uplift pricing for electricity markets," 2003, [online] Available: http://www.hks.harvard.edu/fs/whogan/.

[4] P. R. Gribik, W. W. Hogan and S. L. Pope, "Market-clearing electricity prices and energy uplift," 2007, [online] Available: http://www.hks.harvard.edu/fs/whogan/.

[5] A. L. Motto and F. D. Galiana, "Equilibrium of auction markets with unit commitment: The need for augmented pricing," IEEE Trans. Power Syst., vol. 17, no. 3, pp. 798-805, Aug. 2002.

[6] F. D. Galiana, A. L. Motto and F. Bouffard, "Reconciling social welfare, agent profits and consumer payments in electricity pools," IEEE Trans. Power Syst., vol. 18, no. 2, pp. 452-459, May 2003.

[7] F. Bouffard and F. D. Galiana, "Generalized uplifts in pool-based electricity markets: coordinating time-dynamic markets," in Analysis, Control and Optimization of Complex Dynamic Systems, E. K.Boukas and R. Malhamé, eds., New York, NY: Springer, 2005.

[8] G. Strbac, "Demand side management: Benefits and challenges," Energy Policy, vol. 36, no. 12, pp. 4419-4426, Dec. 2008.

[9] D. S. Kirschen, "Demand-side view of electricity markets," IEEE Trans. Power Syst., vol. 18, no. 2, pp. 520-527, May. 2003.

[10] D. S. Kirschen, G. Strbac, P. Cumperayot and D. de Paiva Mendes, "Factoring the elasticity of demand in electricity prices," IEEE Trans. Power Syst., vol. 15, no. 2, pp. 612-617, May 2000.

[11] D. Papadaskalopoulos and G. Strbac, "Decentralized participation of flexible demand in electricity markets-part I: market mechanism," IEEE Trans. Power Syst., vol. 28, no. 4, pp. 3658-3666, Nov. 2013.

[12] D. Papadaskalopoulos, G. Strbac, P. Mancarella, M. Aunedi and V. Stanojevic, "Decentralized participation of flexible demand in electricity markets-part II: Application with electric vehicles and heat pump systems," IEEE Trans. Power Syst., vol. 28, no. 4, pp. 3667-3674, Nov. 2013.

[13] M. Carrión and J. M. Arroyo, “A computationally efficient mixedinteger linear formulation for the thermal unit commitment problem," IEEE Trans. Power Syst., vol. 21, no. 3, pp. 1371-1378, Aug. 2006.

[14] FICO XPRESS website, http://www.fico.com/en/Products/DMTools/ Pages/FICO-Xpress-Optimization-Suite.aspx. 